\title{
Comparison of computer-assisted planning and manual planning for depth electrode implantations in epilepsy
}

\author{
Mark Nowell, MB, BS, MA, MRCS, ${ }^{1,2}$ Rachel Sparks, PhD, ${ }^{4}$ Gergely Zombori, PhD, ${ }^{4}$ \\ Anna Miserocchi, MD, ${ }^{1-3}$ Roman Rodionov, PhD, ${ }^{1,2}$ Beate Diehl, MD, PhD, ${ }^{1,2,7}$ Tim Wehner, MD, , $, 2,7$ \\ Gianluca Baio, PhD, ${ }^{6}$ Gianluca Trevisi, MD, ${ }^{3}$ Martin Tisdall, MD, FRCS, ${ }^{5}$ Sebastien Ourselin, PhD, ${ }^{4}$ \\ Andrew W. McEvoy, FRCS, ${ }^{1-3}$ and John Duncan, FRCP, FMedSci' ${ }^{1,2}$
}

\begin{abstract}
${ }^{1}$ Epilepsy Society, MRI Unit, Chalfont St Peter; ${ }^{2}$ Department of Clinical and Experimental Epilepsy, University College London Institute of Neurology; ${ }^{3}$ Department of Neurosurgery, National Hospital for Neurology and Neurosurgery; ${ }^{4}$ Centre of Medical Imaging and Computing, University College London; ${ }^{5}$ Department of Neurosurgery, Great Ormond Street Hospital; ${ }^{6}$ Department of Statistical Science, University College London; and 'Department of Clinical Neurophysiology, National Hospital for Neurology and Neurosurgery, London, United Kingdom
\end{abstract}

OBJECTIVE The objective of this study was to evaluate the clinical utility of multitrajectory computer-assisted planning software (CAP) to plan stereoelectroencephalography (SEEG) electrode arrangements.

METHODS A cohort of 18 patients underwent SEEG for evaluation of epilepsy at a single center between August 2013 and August 2014. Planning of electrodes was performed manually and stored using EpiNav software. CAP was developed as a planning tool in EpiNav. The user preselects a set of cerebral targets and optimized trajectory constraints, and then runs an automated search of potential scalp entry points and associated trajectories. Each trajectory is associated with metrics for a safety profile, derived from the minimal distance to vascular structures, and an efficacy profile, derived from the proportion of depth electrodes that are within or adjacent to gray matter. CAP was applied to the cerebral targets used in the cohort of 18 previous manually planned implantations to generate new multitrajectory implantation plans. A comparison was then undertaken for trajectory safety and efficacy.

RESULTS CAP was applied to 166 electrode targets in 18 patients. There were significant improvements in both the safety profile and efficacy profile of trajectories generated by CAP compared with manual planning $(p<0.05)$. Three independent neurosurgeons assessed the feasibility of the trajectories generated by CAP, with 131 (78.9\%) of 166 trajectories deemed suitable for implementation in clinical practice. CAP was performed in real time, with a median duration of 8 minutes for each patient, although this does not include the time taken for data preparation.

CONCLUSIONS CAP is a promising tool to plan SEEG implantations. CAP provides feasible depth electrode arrangements, with quantitatively greater safety and efficacy profiles, and with a substantial reduction in duration of planning within the 3D multimodality framework.

http://thejns.org/doi/abs/10.3171/2015.6.JNS15487

KEY WORDS epilepsy; computer-assisted planning; intracranial EEG; stereoelectroencephalography

$\mathrm{S}$ TEREOELECTROENCEPHALOGRAPHY (SEEG) is the recording of the brain's electrical activity through depth electrodes implanted in the parenchyma of the brain. SEEG is indicated in select patients with pharmacoresistant focal epilepsy to delineate the irritative and seizure onset zones and their relationship to eloquent cortical areas and white matter tracts. ${ }^{5}$ SEEG can be implemented with frame-based and frameless methods of implantation., ${ }^{2,8}$
SEEG planning is a necessary prerequisite to implementation. A number of factors are considered when planning an electrode arrangement for SEEG (Table 1). Individual electrodes should enter at the crown of the gyrus to avoid sulcal boundaries and should avoid vascular structures. Furthermore, the electrode should enter at a reasonable angle perpendicular to the skull to enable the trajectory to be drilled with precision.

ABBREVIATIONS CAP = computer-assisted planning software; IQR = interquartile range; SEEG = stereoelectroencephalography. SUBMITTED March 3, 2015. ACCEPTED June 4, 2015.

INCLUDE WHEN CITING Published online December 4, 2015; DOI: 10.3171/2015.6.JNS15487. 
TABLE 1. Requirements of individual and multiple depth electrodes

\begin{tabular}{lll}
\hline & \multicolumn{1}{c}{ Requirement } \\
\cline { 2 - 3 } Electrode Configuration & \multicolumn{1}{c}{ Absolute } & Relative \\
\hline Individual electrode & & Reasonable entry point on scalp \\
\hline & Avascular plane through brain & Less than $30^{\circ}$ angle of traversing the skull \\
\hline & Accurately hit target & Over 3-mm safety margin to critical structures \\
\hline & Avoid deep sulci at the cortical surface & Maximize number of contacts in gray matter \\
\hline Electrode arrangement & & \\
\hline & Good 3D spatial sampling & Avoid redundant coverage \\
\hline & Avoid electrode collision & \\
\hline
\end{tabular}

Current commercial planning solutions allow the user to select cerebral target and entry points, resulting in the generation of a planned trajectory. The user scrolls along the trajectory in the orthogonal planes, and can also scroll with a "probe's eye" view, which is perpendicular to the trajectory and offers a look-ahead view. In the context of a multitrajectory implantation, this individualized approach is time-consuming and inefficient, with a degree of trial and error necessary to avoid trajectory collision. There is no commercial product that offers computer assistance with planning, to improve either the safety profile or the efficiency of individual trajectories in SEEG.

Previous research in automated planning has centered on deep brain stimulation. ${ }^{1,6}$ Common to all previous work, the operator selects the target point, and a selection of entry points and trajectories are generated, based on the physical constraints entered by the user. There is some evidence that this approach reduces the time taken with planning, and produces clinically feasible electrode trajectories. ${ }^{6}$ The main disadvantage has been that the computation of optimized paths is time-consuming, and not feasible in a clinical context. Recent work has overcome this, achieving real-time speed and using a custom form of visualization (risk maps) to aid the planning process. ${ }^{6}$

The use of computerized automated planning is intuitively more suited to SEEG than to deep brain stimulation, where there is a solitary target and a generally accepted route of approach. SEEG is far more complex, with multiple potential trajectories to any area of the cerebral cortex, with discrete functional and anatomical targets, in patients who may have structural abnormalities in the brain. Additionally, contacts are spaced along the length of each electrode, meaning that the clinician is concerned with the position of the entire trajectory path.

The Milan group reported their experience with an automated planner for use in SEEG, which maximizes the distance of depth electrodes from blood vessels and avoids the sulci as cerebral entry points. This group reported individual depth electrode trajectories, ${ }^{4}$ and multiple trajectories, ${ }^{3}$ describing a quantitative and qualitative validation of the automated multitrajectory planner with 26 electrodes in 3 patients. They concluded that automatic planners are clinically valuable for assisting SEEG planning, and potentially useful for brain biopsy and deep brain stimulation.
We have implemented a 3D multimodal brain-imaging platform (EpiNav [research software not commercially available], CMIC, University College London [UCL]) for epilepsy surgery planning 8,9 and have developed a multitrajectory automated planner for SEEG. We have previously shown the principle of CAP in 6 patients, comprising 52 electrodes, with lower risk values and more contact with gray matter. We have also demonstrated computational efficiency, with a median plan calculation time of 16.68 seconds for 9 trajectories (R. Sparks et al., unpublished data, 2015).

In this paper we describe our comprehensive retrospective study, with an emphasis on clinical validity, to compare the electrode trajectories in 18 patients whose electrode implantations were planned manually and trajectories determined using CAP.

\section{Methods \\ Study Population}

The Joint Research Ethics Committee of the National Hospital for Neurology and Neurosurgery and the UCL Institute of Neurology approved this project. All individuals with medically refractory focal epilepsy, undergoing planning for SEEG between August 2013 and August 2014, were invited to participate in this study and provided informed consent.

\section{Data Preparation}

Patients undergoing planning for SEEG underwent volumetric Gd-enhanced T1-weighted MRI (Siemens Avanto 1.5-T, FOV $512 \times 512 \times 144$, voxel size $0.5 \times 0.5 \times 1.5$ $\mathrm{mm}^{3}$ ), and dedicated vascular imaging in the form of 3D phase-contrast MRI (Siemens Avanto 1.5-T, FOV $256 \times$ $256 \times 160$, voxel size $\left.0.85 \times 0.85 \times 1 \mathrm{~mm}^{3}\right)$, and CT angiography (Siemens Somatom Definition AS, FOV $512 \times$ $512 \times 383$, voxel size $0.43 \times 0.43 .0 .75 \mathrm{~mm}^{3}$ ) in patients in whom high-risk perisylvian trajectories were anticipated.

Image integration and visualization was performed on EpiNav. The T1-weighted MR image was the reference image, upon which other modalities were coregistered. CT angiography and 3D phase-contrast images were processed using vessel extraction software available on EpiNav. ${ }^{12}$ Gray matter, surface sulcal, and cortical segmentations were derived from T1-weighted MRI using 
Freesurfer software (version 5.0.0, Martinos Centre for Biomedical Imaging). Scalp exclusion masks were generated based on the T1-weighted MRI using basic imaging tools in EpiNav and MeshLab (version 1.3.3, University of Pisa). All models were stored and used as surface renderings (Fig. 1).

\section{Imaging Software}

A detailed description of software and the CAP algorithm is given elsewhere ${ }^{11}$ (R. Sparks et al., unpublished data, 2015).

EpiNav is a dedicated platform for image integration, $3 \mathrm{D}$ visualization, and surgical planning in epilepsy surgery. The automated multitrajectory planner calculates optimized electrode configurations in real time, based primarily on reduced cumulative risk scores and within a set of predetermined constraints. The workflow for CAP is as follows: 1) target points set by user; 2) trajectories identified that meet the user-defined constraints of electrode length and angle traversing the skull; 3) trajectories selected where entry points are limited to an individually tailored scalp exclusion mask, which excludes anatomical areas not appropriate for electrode entry such as the face;
4) primary sort of trajectories that optimize the distance from "critical structures," defined as models of surface sulci and cerebral vasculature; and 5) secondary sort of trajectories to optimize the proportion of electrode in gray matter, within an accepted risk profile.

Following planning of electrode trajectories, the user can examine the electrode trajectory, scrolling along the length of the electrode with a "probe's eye viewer," inspecting the minimum distance to the closest defined critical structure, as well as the proximity of gray matter.

For each trajectory, a number of metrics are presented (Fig. 2), as follows: 1) electrode length, the length of the trajectory from the scalp entry point to the cerebral target point; 2) angle, the angle of entry at the skull, where $0^{\circ}$ represents the perpendicular; 3) risk, the cumulative risk across the length of the electrode. This is calculated by taking the area that the electrode resides in the "risk zone," defined as lying between $3 \mathrm{~mm}$ and $10 \mathrm{~mm}$ to the nearest blood vessel. An electrode that never passes within $10 \mathrm{~mm}$ of a blood vessel is given the average risk value of 0 . An electrode that passes to within $3 \mathrm{~mm}$ is automatically given an average risk value of 1 , since $3 \mathrm{~mm}$ is the accuracy of delivery of the electrode using the frameless SEEG $\left.\operatorname{method}^{8}\right)$; 4) minimum distance, the lowest distance along
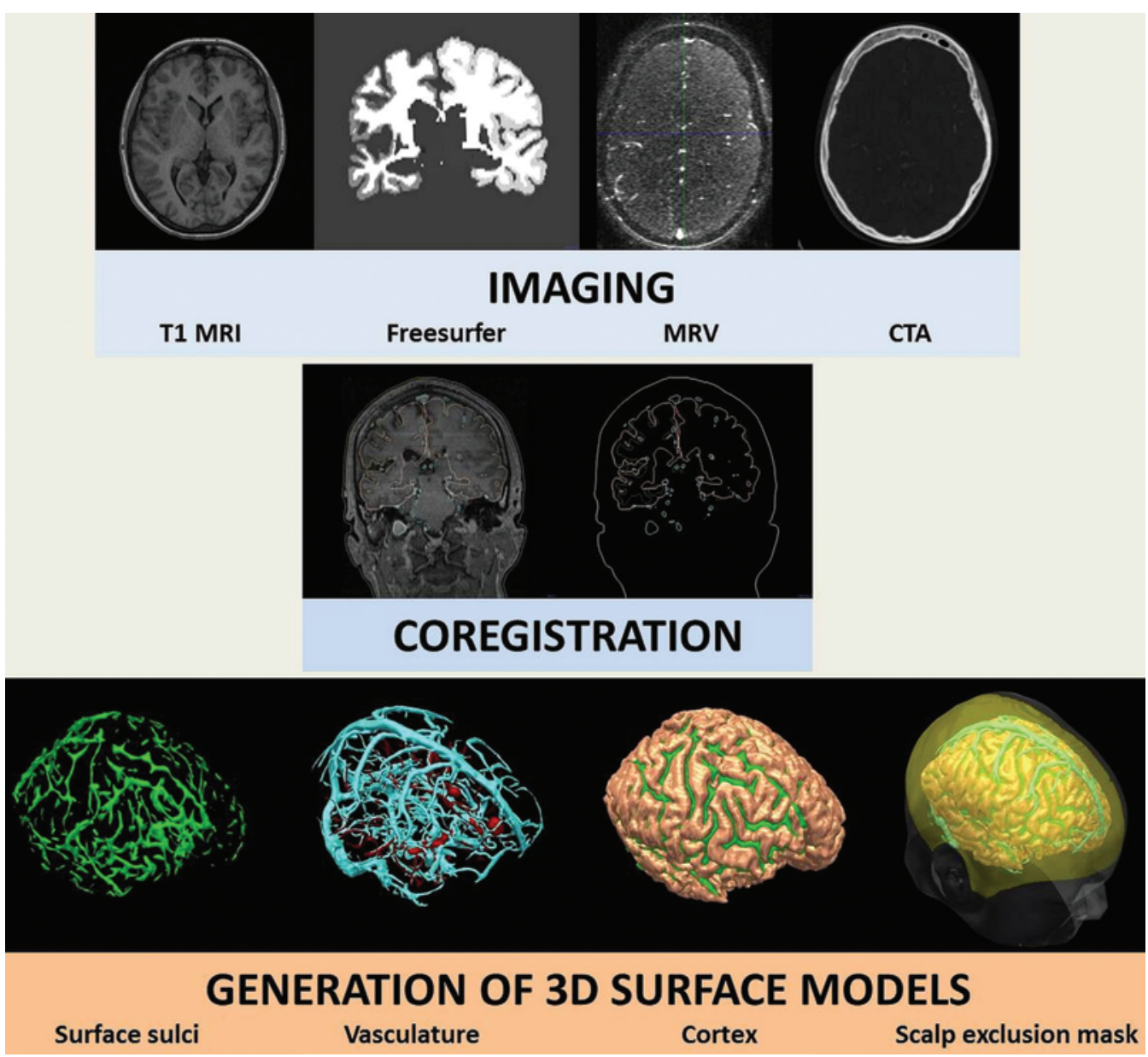

FIG. 1. Data preparation for computer-assisted planning. Stages include image acquisition, coregistration, and then 3D surface rendering. MRV = MR venography. 


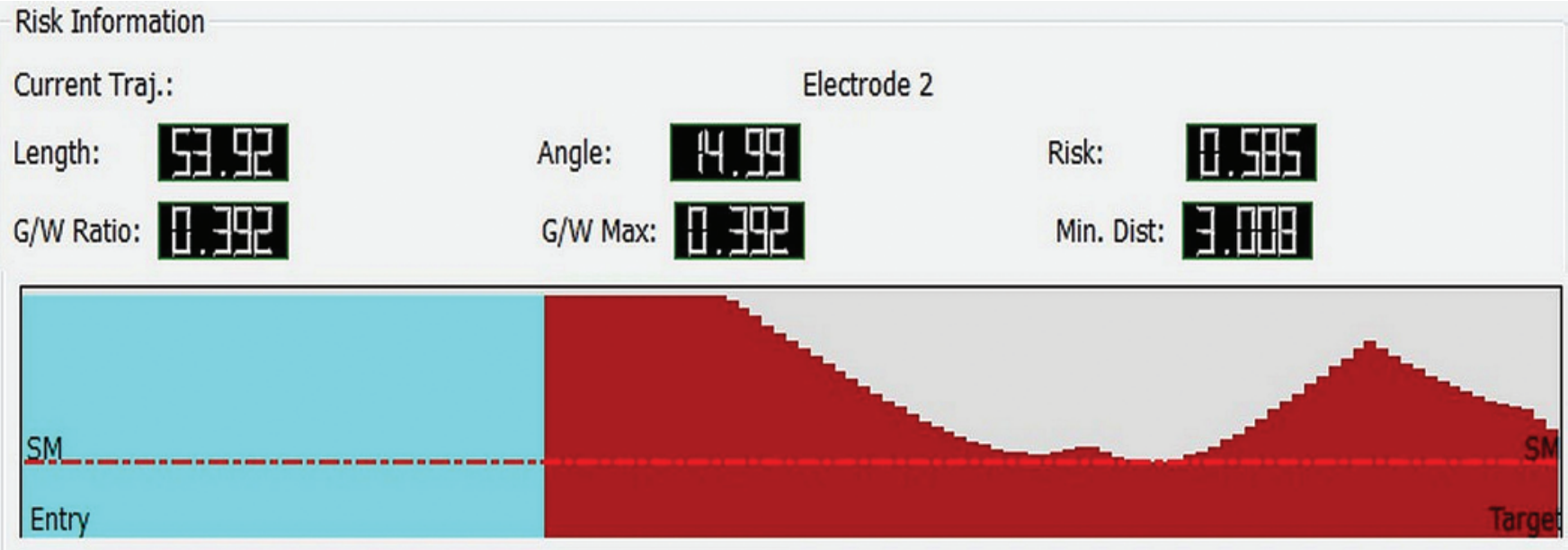

FIG. 2. Graphic visualization of metrics associated with individual trajectories. Upper: Length, angle traversing skull, risk, gray/ white $(\mathrm{G} / \mathrm{W})$ ratio, and minimum distance from a blood vessel $>1 \mathrm{~mm}$ in diameter. Center: Graphic display of closest critical structure along length of trajectory (red = artery, cyan = vein, y-axis = distance to structure [maximum $10 \mathrm{~mm}$ ], $\mathrm{x}$-axis = distance along trajectory from brain entry to target, $\mathrm{SM}=$ safety margin represented as horizontal red line that marks 3-mm separation of trajectory to critical structure). Lower: Graphic display of trajectory path through gray and white matter (green $=$ extracerebral, gray $=$ gray matter, white $=$ white matter).

the length of the trajectory to the nearest blood vessel, expressed in $\mathrm{mm}$; and 5) gray to white matter ratio, defined as the proportion of the electrode from the cortical surface to the cerebral target point that lies within gray matter. This is a reflection of the efficacy profile of the electrode, because recordings from gray matter are of greater utility than recordings from white matter when determining the source of epileptic activity.

\section{Manual Planning}

Previously, each case had been discussed at a multidisciplinary team meeting, involving neurologists, neurophysiologists, neuroradiologists, neuropsychologist, psychiatrists, and neurosurgeons. Targets for depth electrode coverage were agreed upon, and manual planning of the implantation had taken place on EpiNav using conventional techniques for individual electrodes. These manual plans were stored on a single workstation, following implementation in the operating theater. With the development of CAP, the manual plans were reexamined, extracting the quantitative metrics of electrode length, angle of entry, risk, minimum distance, and gray to white matter ratio.

\section{CAP Software}

The 3D target coordinates were reentered into a new plan, and CAP on EpiNav was run to derive a new automated plan, with new associated metrics for individual electrodes. The initial user constraints used were electrode length $80 \mathrm{~mm}$ and entry angle within $15^{\circ}$ of perpendicular to the skull, with a minimum distance of $1 \mathrm{~cm}$ between trajectories. These constraints were selected to generate trajectories with short intracerebral lengths that would be straightforward to drill and implement in the theater.
Suitable individual trajectories were "locked," and CAP was rerun with adjusted user constraints for the remaining electrodes. In this way, an electrode configuration was generated that could accommodate differential requirements for individual electrodes.

\section{Feasibility of Implementation}

All manual planning is feasible, as these plans had already been implemented in clinical practice. The feasibility of implementation of the results of CAP was assessed by 3 epilepsy neurosurgeons independently using the EpiNav software. Each electrode trajectory was examined for scalp and cortical entry points using 3D computer models, and avascular paths were checked using the probe's eye viewer. The trajectory was then given a pass or fail in terms of feasibility of clinical implementation. If twothirds of the neurosurgeons "passed" a trajectory, it was considered clinically feasible.

In addition, the neurosurgeon was presented with the overall electrode arrangement derived from manual planning and CAP in a blinded way, and was asked to determine feasibilities of entire implantations (Fig. 3). One of the 3 neurosurgeons was involved in both manual planning and CAP. However, CAP was 3-15 months after the prior manual planning, and we do not believe this introduced any bias into the study.

\section{Results}

Eighteen patient implantations were studied, comprising 166 electrodes, with a range of 6-12 per patient. Demographics of the patient group are described in Table 2.

All manual planning had previously been implemented in clinical practice. In 11 of 18 patients a putative epi- 

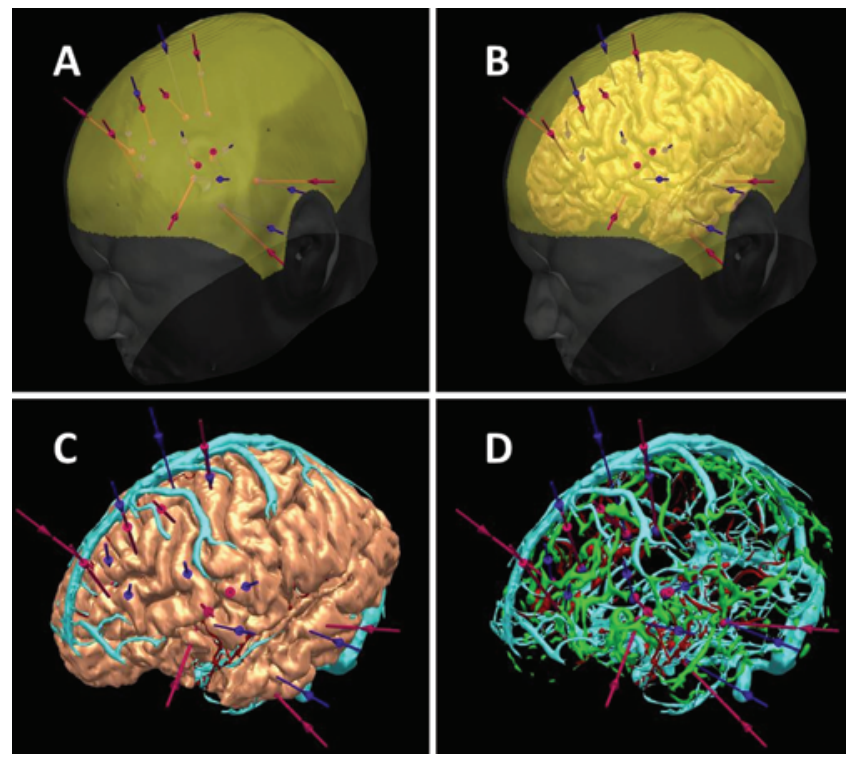

FIG. 3. 3D visualization of manual (blue) versus CAP (purple) in an individual patient. A: Scalp (white), scalp exclusion mask (yellow). B: Brain added (pink). C: Scalp and mask removed, and veins (cyan) add-

ed. D: Brain removed and arteries (red) and surface sulci (green) added.

leptogenic zone was identified following SEEG that was amenable to resection, and these patients proceeded to resective surgery. In 4 of 18 patients, the putative epileptogenic zone was identified, but cortical resection was not continued due to the risk of neurological deficit. The implantation failed to localize the epileptogenic zone in 2 patients, and was prematurely terminated in 1 patient on request before an adequate duration of recording. There was 1 hemorrhagic complication, which occurred on removal of electrodes. This complication resulted in a mild temporary upper-limb weakness and did not require any surgical intervention.

CAP generated $163(98.2 \%)$ of 166 trajectories. There were 3 cerebral targets for which no trajectory was found that satisfied the user constraints, including 2 orbitofrontal targets and 1 anterior hippocampal target.

\section{Metrics}

A comparison of the results of manual planning versus CAP is demonstrated in Table 3 and Fig. 4. We assessed the differences accounting for the correlation induced by the repeated measurements for each patient, using multilevel models with a varying (patient-specific) intercept.

Manual planning resulted in a median electrode length of $57.9 \mathrm{~mm}$ (interquartile range [IQR] $21.8 \mathrm{~mm}$ ) and a median angle of traversing the skull of $16.2^{\circ}$ (IQR $12.9^{\circ}$ ) off the perpendicular. CAP resulted in a median electrode length of $53.9 \mathrm{~mm}$ (IQR $15.6 \mathrm{~mm}$ ) and a median angle of traversing the skull of $13.0^{\circ}$ (IQR $7.6^{\circ}$ ) off the perpendicular. These differences are likely to be due to the angle of entry being a constraining factor in CAP, generating more perpendicular and consequently shorter electrode lengths.

Manual planning resulted in a median risk value of 0.41 (IQR 0.79), with a median minimum distance to a critical structure of $4.48 \mathrm{~mm}$ (IQR $2.99 \mathrm{~mm}$ ) and a median gray to white matter ratio of 0.33 (IQR 0.33 ). CAP resulted in a median risk value of 0.36 (IQR 0.42 ) with a median minimum distance to a critical structure of $4.52 \mathrm{~mm}$ (IQR 2.97 $\mathrm{mm}$ ) and a median gray to white matter ratio of 0.48 (IQR 0.28).

Applying standard regression models with random effect, there was a significant difference between man-

TABLE 2. Demographics of the patient study group and the time taken for CAP

\begin{tabular}{rrlrlr}
\hline Case No. & Age (yrs), Sex & Presumed EZ & No. of Electrodes & Vascular Models & Duration of CAP (min) \\
\hline 1 & $29, \mathrm{M}$ & Lt frontal & 13 & Veins & 12 \\
\hline 2 & $29, \mathrm{M}$ & Lt frontal & 10 & Veins, arteries & 8 \\
\hline 3 & $19, \mathrm{~F}$ & Rt occipital & 9 & Veins, arteries & 12 \\
\hline 4 & $27, \mathrm{M}$ & Rt parietal & 10 & Veins & 6 \\
\hline 5 & $40, \mathrm{M}$ & Rt occipital & 11 & Veins, arteries & 7 \\
\hline 6 & $32, \mathrm{M}$ & Rt temporal & 7 & Veins, arteries & 16 \\
\hline 7 & $19, \mathrm{M}$ & Rt frontal & 7 & Veins, arteries & 3 \\
\hline 8 & $37, \mathrm{~F}$ & Rt temporal & 7 & Veins & 9 \\
\hline 9 & $29, \mathrm{M}$ & Rt temporal & 12 & Veins & 19 \\
\hline 10 & $31, \mathrm{M}$ & Lt frontal & 11 & Veins, arteries & 13 \\
\hline 11 & $42, \mathrm{~F}$ & Rt frontal & 8 & Veins, arteries & 5 \\
\hline 12 & $22, \mathrm{~F}$ & Rt parietal & 8 & Veins & 6 \\
\hline 13 & $37, \mathrm{M}$ & Rt insula & 11 & Veins arteries & 8 \\
\hline 14 & $32, \mathrm{~F}$ & Lt temporal & 7 & Veins, arteries & 4 \\
\hline 15 & $26, \mathrm{M}$ & Rt frontal & 8 & Veins, arteries & 7 \\
\hline 16 & $44, \mathrm{M}$ & Rt frontal & 7 & Veins, arteries & 9 \\
\hline 17 & $61, \mathrm{~F}$ & Rt temporal & 8 & Veins, arteries & 12 \\
\hline 18 & $42, \mathrm{M}$ & Rt temporal & 12 & &
\end{tabular}


TABLE 3. Statistical comparison between manual planning and CAP

\begin{tabular}{lccrrr}
\hline \multicolumn{1}{c}{ Measurement } & Manual Planning* & CAP* & $\begin{array}{c}\text { Estimated Difference } \\
\text { (Manual - CAP) }\end{array}$ & Error & $p$ Value \\
\hline Electrode length (mm, 1dp) & $57.9(21.8)$ & $53.9(15.6)$ & 4.74 & 1.59 & $<0.05$ \\
\hline Angle of entry ( ${ }^{\circ}$ off perpendicular, 1dp) & $16.2(12.8)$ & $13.0(7.6)$ & 5.89 & 1.07 & $<0.05$ \\
\hline Risk (normalized units, 2dp) & $0.41(0.79)$ & $0.36(0.42)$ & 0.19 & 0.03 & $<0.05$ \\
\hline Minimum distance from blood vessel (mm, 1dp) & $4.5(3.0)$ & $4.5(3.0)$ & -0.56 & 0.20 & $<0.05$ \\
\hline Proportion of intracerebral electrode in gray matter (2dp) & $0.33(0.33)$ & $0.48(0.28)$ & -0.11 & 0.02 & $<0.05$ \\
\hline
\end{tabular}

$\mathrm{dp}=$ decimal place.

* Values are median (IQR).

ual planning and CAP in terms of all metrics measured (Table 3). CAP generates shorter trajectories that traverse the skull closer to the perpendicular, with lower risk values, greater minimum distances to critical structures, and higher gray to white matter ratios.

CAP was better at avoiding high-risk trajectories, defined as those that pass within $3 \mathrm{~mm}$ of a critical structure, giving a risk value of 1 or above. This occurred in 44 trajectories with manual planning, and in only 9 cases in CAP.

\section{Feasibility}

Individual CAP trajectories in 131 (78.9\%) of 166 were deemed suitable for clinical implementation without adjustment, in terms of appropriate scalp and cortical entry points and avascular paths, following independent review by 3 neurosurgeons. Thirty-five trajectories required manual adjustment (Table 4). This was most commonly because of the proximity to blood vessels that were evident on the probe's eye view projection but not represented

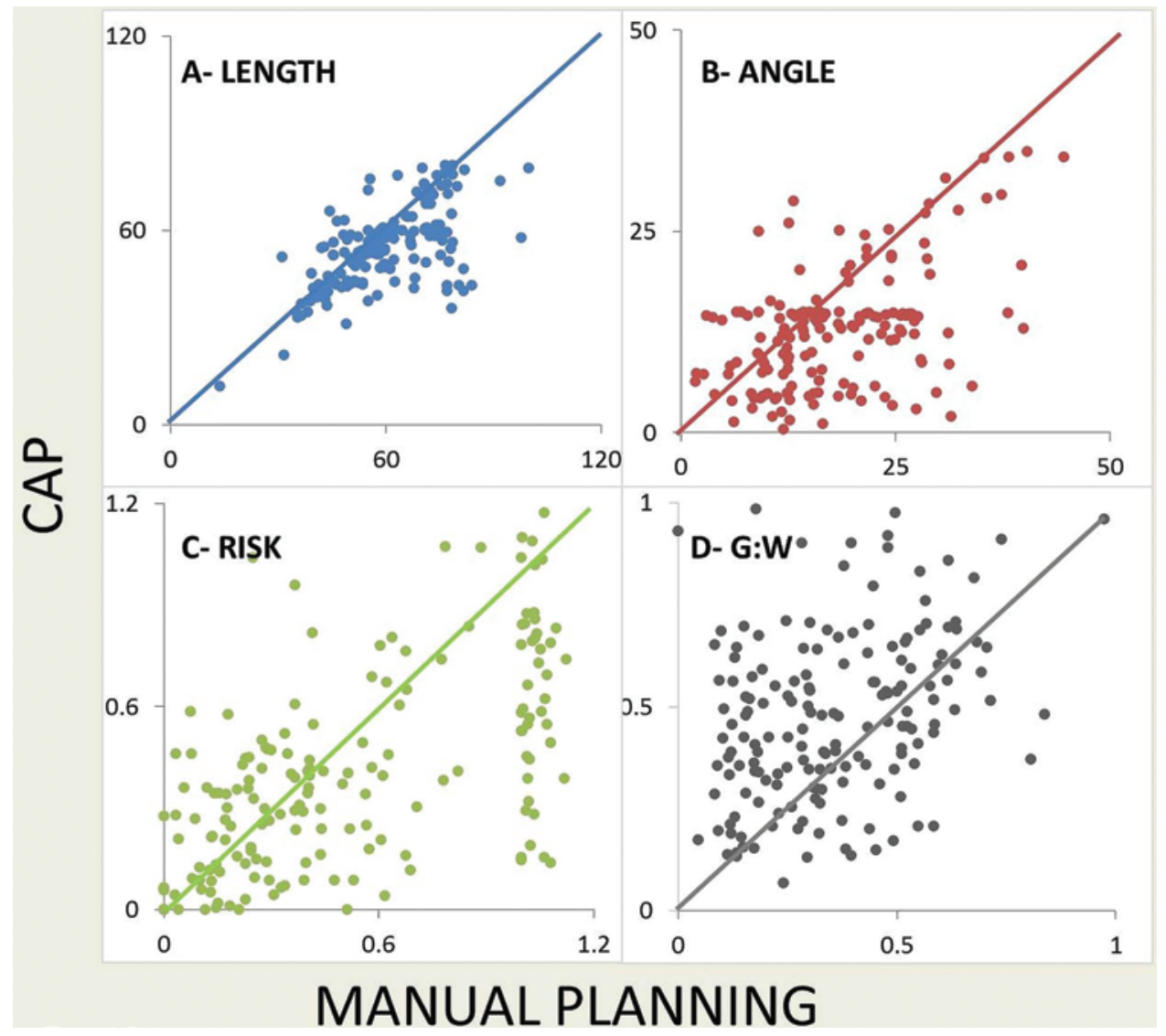

FIG. 4. Comparison of manual planning versus CAP for electrode length $(\mathbf{A})$, angle of entry $(\mathbf{B})$, risk $(\mathbf{C})$, and gray-white matter ratio (G:W; D). In each graph, the $x$-axis represents manual planning and the $y$-axis represents computer-assisted planning. Each point represents the value of the given metric for manual planning and CAP. Any point located below the diagonal line means that the value derived from manual planning is greater than that from CAP and vice versa. For $A, B$, and $C$, values below the line indicate superiority of CAP over manual planning. For $\mathrm{D}$, values above the line indicate superiority of CAP over manual planning. 
as surface-rendered structures due to suboptimal segmentation. Work is ongoing to improve the quality of vessel segmentation. All 18 (100\%) of 18 implantations planned using CAP were deemed to be feasible for clinical implementation.

\section{Timing}

The prior manual SEEG planning typically took 2-3 hours per patient, although the duration was not prospectively recorded, and we recognize this as a limitation of our study. The duration of CAP is recorded in Table 2. The median duration of CAP was 8 minutes. This includes the generation of the trajectories as well as the checking of the trajectories with the 3D models, the risk visualization graph, and the probe's eye viewer. Duration was largely dependent on the number of "rounds" of CAP that were needed to satisfy individual trajectory constraints. However, this does not take into account the time taken for data preparation (generation of scalp exclusion mask and models of sulci and gray matter), which can take a total of 20-30 minutes per patient. Additionally, this does not take into account the time required for the manual adjustment of trajectories that were not considered feasible, which takes approximately 2-3 minutes per electrode.

\section{Discussion}

\section{Summary}

The process of manual planning in SEEG is time consuming and complex, with the dual aims of achieving good coverage of the putative epileptogenic zone and network, and adjacent eloquent cortex, and ensuring safe, practical trajectories. We previously demonstrated that planning is assisted by the use of 3D multimodality imaging, demonstrating the spatial relationships between planned trajectories, structural and functional regions of interest, gyral anatomy, and cortical vasculature. , $^{10}$

We describe CAP, which has been tested on 18 historical cases comprising 166 depth electrodes. CAP provides rapid, optimized solutions for electrode configurations, with statistically significant improvements in risk value and gray/white matter capture compared with conventional manual planning, and with substantial savings in time within the framework of a 3D multimodality model.

\section{Data Preparation}

For CAP to be effective, good data preparation is paramount. Individually tailored scalp exclusion masks are needed to avoid inappropriate skull entry points on the face, ears, below the tentorium cerebelli, and crossing the midline. The use of surface sulcal models is important to center the electrodes on the crown of the gyri, although it is acknowledged that some surgical groups accept crossing sulcal boundaries. Most importantly, the quality of the risk values is dependent on quality of the segmentations of vascular structures. In this series, the most common reason for trajectories being rejected for clinical implementation was proximity to blood vessels that were not included in the surface models. Complete vessel segmentation is difficult to achieve without invasive cerebral angiography, which adds considerably to the patient workup and preoperative
TABLE 4. The causes for CAP electrode trajectories that required manual adjustment

\begin{tabular}{lc}
\hline \multicolumn{1}{c}{ Reason for Classification of Failed Trajectory } & Value \\
\hline Proximity to blood vessel (missing from 3D vessel model) & 18 \\
\hline Proximity to blood vessel (included in 3D vessel model) & 5 \\
\hline Proximity to superior sagittal sinus & 6 \\
\hline Proximity to deep sulcus & 4 \\
\hline Entry point not feasible & 2 \\
\hline Total & 35 \\
\hline
\end{tabular}

risk. Our group continues to work on this problem, with possible roles for center-lining vessel structures, derived from multiple sources of noninvasive vascular imaging, and dual-energy $\mathrm{CT}$ angiography.

Data preparation takes time. In our experience, complete 3D multimodality image integration for any patient using EpiNav takes 20-30 minutes, and this does not take into account the postprocessing performed using thirdparty software. In our center, it is routine for all patients undergoing an intracranial EEG study to have 3D multimodality image integration performed, as we have previously demonstrated its clinical utility in decision making. ${ }^{9}$ We would therefore not consider data preparation as an additional step in our own workflow, although this may not be true in other centers and should be factored into any proposed time savings.

\section{Risk Reduction}

In this series, the reduction of risk from CAP is reflected in the reduced risk value. It is important to acknowledge that this value is representative of cumulative risk over the length of the electrode. As an absolute value, this is of limited use to the clinician, as it is heavily affected by the critical structures presented. Risk cannot be compared across different patients, because the quality of image acquisition and segmentation varies. However, the value is most useful for comparing CAP with manual planning in a single patient with the same critical structure segmentation. In contrast, "least distance to the nearest critical structure" is an easy metric to understand. In this series, there was a significant increase in this metric when using CAP, giving further evidence that CAP improves the safety profile of implantations. However, the translation of this metric into actual reduction in hemorrhage rate is difficult to demonstrate in this small series.

\section{Increasing Gray Matter Capture: Making Electrodes Work Harder}

With manual planning, the median proportion of trajectories in gray matter was 0.33 , compared with 0.48 in CAP. It is intuitive that increasing the proportion of electrodes in gray matter improves the chances of siting individual contacts in gray matter, with a corresponding improvement in the 3D capture of epileptic activity and neurophysiological "yield," although this was not demonstrated in this study.

The next step to improve efficacy is to model electrode contacts in the planning stage, with the aim of siting 
these in gray matter segmentations. This would reduce the "waste" of redundant contacts that lie in white matter. For this to be effective, a very precise method of SEEG implementation will be required, with 1 - to $2-\mathrm{mm}$ target point precision.

\section{Feasibility}

The feasibility study performed by 3 neurosurgeons on the CAP implantations demonstrated 2 findings. First, individual electrode trajectories generated with CAP were generally believed to be safe and feasible for clinical implementation. A small proportion of trajectories had to be revised on account of failures of vessel segmentation, and a small number of trajectories were rejected because of their proximity to segmented vessels and deep sulci. This reinforces the caveat that all trajectories need to be thoroughly cross-checked using the probe's eye view with conventional imaging before they are accepted for clinical implementation.

Second, the overall trajectory configurations generated were also feasible for clinical implementation. A given constraint to CAP is that trajectories are spaced by at least $1 \mathrm{~cm}$. However, at present there is no functionality to space the trajectories in an aesthetically pleasing and more even fashion on the cortical surface. Further, there is no weighting of the vascular surface renderings, with a consequence that CAP makes no distinction between large vascular structures (i.e., the superior sagittal sinus) and smaller surface veins. This could be addressed in the future by generating a more limited scalp exclusion mask that excludes a longitudinal strip along the midline.

Perhaps the strongest limitation to this feasibility study is that it only examines how to safely design trajectories to a target point, with little emphasis on the importance of the cortical entry point and traversing gray matter. Because the entire length of the trajectory is important to the capture of epileptic activity, it is possible that the CAP configurations are less effective at capturing desired superficial cortical EEG activity. These limitations will be resolved by further constraining the target entry zones of specific electrodes, making CAP trajectories not just feasible but optimal for delineating the epileptogenic zone.

A cortical parcellation library of the patient's individual brain would facilitate the design of efficient electrode trajectories that sample both superficial convexity cortex and deep targets that are of interest, and would also allow for differential electrode constraints in a single "application" of CAP; for example, an entry point in the left anterior superior frontal gyrus and a target in the anterior left insula, which would present a very constrained number of possible trajectories, and would be more efficient computationally. This would also give the user more control in designing an evenly spaced trajectory configuration that does not run close to large vascular structures. Our group is currently working toward incorporating this into our version of CAP.

\section{Clinical Utility}

A pertinent question raised by this study is whether the gains that are described translate into true clinical utility in actual practice, in terms of fewer complications and better determination of the epileptogenic zone. Randomized controlled trials are notoriously difficult to perform in epilepsy surgery due to the heterogeneity of the patient group. Our group plans to incorporate CAP into a prospective SEEG planning study, with the addition of a cortical parcellation library and more complete vascular segmentations, where the surgeon uses CAP as a starting point to the planning process, prior to manual checking and adjustment.

In the future, it is possible that CAP may establish certain "corridors" for defined trajectories, based on feasibility studies and these metrics of safety and risk. It is attractive to then consider a "menu" of trajectory implantations that are selected "off the shelf" and that undergo minor adjustments tailored to that individual patient's anatomy. This development would have the benefits of safety, efficiency, and time saving, as well as standardizing SEEG implantation across different centers, making for easier clinical and research collaboration.

\section{Conclusions}

CAP is a promising tool to plan SEEG implantations. CAP provides feasible depth electrode arrangements, with quantitatively greater safety and efficacy profiles, and with substantial savings in duration of planning. Furthermore, CAP has possible uses beyond SEEG, in the planning of the insertion of any hardware into the brain. As minimally invasive treatments become more popular in neurosurgery, safe planning tools that increase safety and efficacy will become increasingly important.

\section{Acknowledgments}

We are grateful to the Wolfson Trust and the Epilepsy Society for supporting the Epilepsy Society MRI scanner. This work was supported by the National Institute for Health Research (NIHR) University College London Hospitals Biomedical Research Centre (BRC). Mark Nowell, Gergely Zombori, Rachel Sparks, and Roman Rodionov are supported by the Department of Health and Wellcome Trust through the Health Innovation Challenge Fund (HICF-T4-275, Programme Grant 97914) and are working in collaboration with Medtronic, Inc., on a project entitled "Novel Multimodality Imaging in Epilepsy Surgery."

\section{References}

1. Brunenberg EJ, Vilanova A, Visser-Vandewalle V, Temel Y, Ackermans L, Platel B, et al: Automatic trajectory planning for deep brain stimulation: a feasibility study. Med Image Comput Comput Assist Interv 10:584-592, 2007

2. Cardinale F, Cossu M, Castana L, Casaceli G, Schiariti MP, Miserocchi A, et al: Stereoelectroencephalography: surgical methodology, safety, and stereotactic application accuracy in 500 procedures. Neurosurgery 72:353-366, 2013

3. De Momi E, Caborni C, Cardinale F, Casaceli G, Castana L, Cossu M, et al: Multi-trajectories automatic planner for StereoElectroEncephaloGraphy (SEEG). Int J Comput Assisted Radiol Surg [epub ahead of print], 2014

4. De Momi E, Caborni C, Cardinale F, Castana L, Casaceli G, Cossu M, et al: Automatic trajectory planner for StereoElectroEncephaloGraphy procedures: a retrospective study. IEEE Trans Biomed Eng 60:986-993, 2013

5. Duncan JS: Selecting patients for epilepsy surgery: synthesis of data. Epilepsy Behav 20:230-232, 2011

6. Essert C, Haegelen C, Jannin P: Automatic computation of 
electrodes trajectory for deep brain stimulation, in Liao $\mathrm{H}$, Edwards PJ, Pan X, et al (eds): Lecture Notes in Computer Science 6326: Medical Imaging and Augmented Reality. Berlin: Springer, 2010, 149-158

7. Nowell M, Miserocchi A, McEvoy AW, Duncan JS: Advances in epilepsy surgery. J Neurol Neurosurg Psychiatry 85:1273-1279, 2014

8. Nowell M, Rodionov R, Diehl B, Wehner T, Zombori G, Kinghorn J, et al: A novel method for implementation of frameless StereoEEG in epilepsy surgery. Neurosurgery 10 (Suppl 4):525-534, 2014

9. Nowell M, Rodionov R, Zombori G, Sparks R, Winston G, Kinghorn J, et al: Utility of 3D multimodality imaging in the implantation of intracranial electrodes in epilepsy. Epilepsia 56:403-413, 2015

10. Rodionov R, Vollmar C, Nowell M, Miserocchi A, Wehner T, Micallef C, et al: Feasibility of multimodal 3D neuroimaging to guide implantation of intracranial EEG electrodes. Epilepsy Res 107:91-100, 2013

11. Zombori G, Rodionov R, Nowell M, Zuluaga MA, Clarkson MJ, Micallef C, et al: A computer assisted planning system for the placement of sEEG electrodes in the treatment of epilepsy, in Stoyanov D, Collins DL, Sakuma I, et al (eds): Lecture Notes in Computer Science 8498: Information Processing in Computer-Assisted Interventions. Cham, Switzerland: Springer, 2014, 118-127

12. Zuluaga MA, Rodionov R, Nowell M, Achhala S, Zombori G, Cardoso MJ, et al: SEEG trajectory planning: combining stability, structure and scale in vessel extraction, in Golland P, Hata N, Barillot C, et al (eds): Medical Image Computing and Computer-Assisted Intervention-MICCAI 2014. Cham, Switzerland: Springer, 2014, pp 651-658

\section{Disclosures}

This publication presents independent research supported by the
Health Innovation Challenge Fund (HICF-T4-275, Programme Grant 97914), a parallel funding partnership between the Department of Health and Wellcome Trust. The views expressed in this publication are those of the authors and not necessarily those of the Department of Health or Wellcome Trust. John Duncan has received institutional grant support from Eisai, UCB Pharma, GSK, Janssen Cilag, Medtronic, and GE Healthcare. Andrew McEvoy has received support from UCB, Baxter, and Cyberonics.

\section{Author Contributions}

Conception and design: Nowell, Sparks, Zombori, Rodionov, Duncan. Acquisition of data: Nowell, Miserocchi, Ourselin. Analysis and interpretation of data: Nowell, Baio, Trevisi, Tisdall. Drafting the article: Nowell. Critically revising the article: Nowell, Sparks, Zombori, Miserocchi, Rodionov, Diehl, Wehner, Baio, Tisdall, Duncan. Reviewed submitted version of manuscript: Nowell, Sparks, Zombori, Miserocchi, Rodionov, Diehl, Wehner, Tisdall, Ourselin, Duncan. Approved the final version of the manuscript on behalf of all authors: Nowell. Administrative/technical/material support: Nowell, Sparks, Zombori, Rodionov, Diehl, Wehner. Study supervision: Diehl, Wehner, Ourselin, Duncan.

\section{Supplemental Information Previous Presentations}

Portions of this work have been presented as a platform presentation at the European Association of Neurosurgeons, in Prague, October 15, 2014, and as an abstract at the Information Processing in Computer-Assisted Interventions, in Fukuoka, Japan, June 28,2014 .

\section{Correspondence}

Mark Nowell, Box 29, National Hospital for Neurology and Neurosurgery, Queen Square, London WC1N 3BG, United Kingdom. email: m.nowell@ucl.ac.uk. 